\title{
A Note on the Convergence to Competitive Equilibria in Economies with Moral Hazard
}

\author{
A. Bisin \\ New York University \\ P. Gottardi \\ Universita' di Venezia and Yale University \\ D. Guaitoli \\ Universitat Pompeu Fabra and C.E.P.R.
}

December $1998^{*}$

\footnotetext{
*the C.V. Starr Center for Applied Economics; CNR and MURST; the Spanish DGICYT.
} 


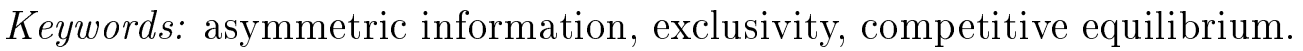
JEL: D82ГD50. 


\title{
A Note on the Convergence to Competitive Equilibria in Economies with Moral Hazard
}

\begin{abstract}
We examine the conditions under which competitive equilibria can be obtained as the limit when the number of strategic traders gets large of Nash equilibria in economies with asymmetric information on agents' effort and possibly imperfect observability of agents' trades. Convergence always occur when either effort is publicly observed (no matter what is the information available to intermediaries on agents' trades); or effort is private information but agents' trades are perfectly observed; or no information at all is available on agents' trades. On the other hand $\Gamma$ when each intermediary can observe its trades with an agent but not the agent's trades with other intermediaries $\Gamma$ the (Nash) equilibria with strategic intermediaries do not converge to any of the competitive equilibria $\mathrm{ffor}$ an open set of economies. The source of the difficulties for convergence is the combination of asymmetric information and the restrictions on the observability of trades which prevent the formation of exclusive contractual relationships and generate barriers to entry in the markets for contracts.
\end{abstract}




\section{Introduction}

After the initial contributions of Radner (1968) and Prescott-Townsend (1984) $\Gamma$ the analysis of competitive equilibria of economies with asymmetric information has recently received renewed attention. For such economies the interaction between the private information dimension (e.g. the unobservable action in the moral hazard case) and the observability of agents' trades plays a crucial role $\Gamma$ since trades have typically informational content over the agents' private information. In particular t to decentralize incentive efficient Pareto optimal allocations the availability of fully exclusive contracts $\Gamma$ i.e. of contracts whose terms (price and payoff) depend on the transactions in all other markets of the agent trading the contract $\Gamma$ is generally required. The implementation of these contracts imposes typically the very strong informational requirement that all trades of an agent need to be observed.

We do observe though agents engaging in different contractual relationships (e.g. having loans both from banks and credit card companies Tholding various insurance policies $[.$. .). It is then of interest to analyze also situations where contracts traded are necessarily non-exclusive $\Gamma$ because perfect monitoring of trades is not available. The case of complete anonymity of trades $\Gamma$ where no transaction of the agents is observable $\Gamma$ constitutes an important benchmark in this respect.

In the framework of a Walrasian competitive equilibrium model $\Gamma$ alternative assumptions on the observability of agents' trades may be captured $\Gamma$ in a somewhat reduced form $\Gamma$ by alternative assumptions on the possible non-linearities of equilibrium prices. The complete observability of trades (exclusivity) is captured by allowing price schedules to be arbitrary nonlinear functions of agents' trades. At the other extreme $\Gamma$ complete anonimity of trades (full non-exclusivity) corresponds to restricting price schedules to be a linear function of trades. Close to this is the case in which only short and long trading positions can be distinguished $\Gamma$ and is captured by price schedules characterized by distinct bid and ask prices.

The existence of competitive equilibria with linear prices has been recently investigated by DubeyГGeanakoplos and Shubik (1995) ГBisin and Gottardi

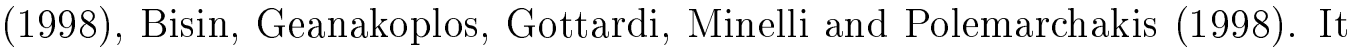

is shown that $\Gamma$ with asymmetric information $\Gamma$ a minimal form of non-linearity $\Gamma$ i.e. the possibility of having a different price for buyers and sellers (a bid-ask spread) is necessary (in fact also sufficient) for competitive equilibria to exist. 
Existence of competitive equilibria with general non-linear price schedules for asymmetric information economies has been studied by Prescott-Townsend (1984). ${ }^{1}$

To better evaluate the informational requirements and the structure of markets implicit in these competitive equilibrium notions examine the conditions under which such equilibria can be obtained as the limit $\Gamma$ as the number of strategic traders gets large $\Gamma$ of the Nash equilibria of a game (where information and strategy sets are explicitly modelled). In this paper we consider a simple economy with moral hazard (i.e. where agents undertake a possibly unobservable action) $\Gamma$ and examine whether $\Gamma$ for different assumptions about the observability of agents' trades $\Gamma$ the (Nash) equilibria of the economy where strategic financial intermediaries compete by issuing contracts converge $\Gamma$ as the number of intermediaries gets large $\Gamma$ to the competitive equilibria of the economy.

With symmetric information the equilibria of the model with strategic intermediaries always converge $\Gamma$ no matter what is the information available to intermediaries over agents' trades $\Gamma$ to competitive equilibria with linear prices. In the presence of asymmetric information $\Gamma$ with complete observability of trades (i.e. when intermediaries are able to implement exclusive contracts) convergence also holds: the Nash equilibria converge $\Gamma$ in this case to the (incentive efficient) competitive equilibria with fully non-linear prices ${ }^{2}$. On the other hand $\Gamma$ when information over agents' trades is more limited $\Gamma$ convergence is not always ensured.

We consider in particular the case in which each intermediary cannot observe any of the trades agents make with other intermediaries $\Gamma$ nor whether agents engage in re-trading fractions of the contracts they purchased (or sold) from them. Intermediaries are only able to monitor the transactions each agent makes with them; hence the only restrictions they can implement are restrictions preventing agents from buying multiple units of the contracts they issue. In such a situation while $\Gamma$ as we already argued $\Gamma$ when agents' effort is commonly observed (i.e. information is symmetric) equilib-

\footnotetext{
${ }^{1}$ More precisely, in Prescott-Townsend (1984) the specification of agents' budget sets restricts admissible trades to lie in the set of incentive compatible trades, which is equivalent in their set-up to allowing general non-linear price schedules; see also Lisboa (1996).

${ }^{2}$ The incentive efficiency of equilibria with competing intermediaries, when exclusive contracts are available, has been shown by Bennardo and Chiappori (1998) for an economy with moral hazard which include as a special case the one considered here.
} 
ria converge to competitive equilibria with linear prices $\Gamma$ the same is not true with asymmetric information. More precisely एwe will show that there exists an open set of parameter values describing the economy for which we do not have convergence: the (Nash) equilibria with strategic intermediaries do not converge to any of the competitive equilibria with bid-ask spreads (i.e. to the competitive equilibria exhibiting the informationally least demanding form of non-linearity which ensures existence). In other words $\Gamma$ the ability of intermediaries to prevent agents from buying multiple units of the contracts they issue interacts with the asymmetry of information (the unobservability of agents' effort choices) to generate barriers to entry in the markets for contracts. Thus the source of the difficulties for convergence is the combination of the presence of asymmetric information and the restrictions on the observability of trades which prevent the formation of exclusive contractual relationships.

Finally $\Gamma$ we show that convergence to competitive equilibria with bidask spreads again obtains if we assume that each intermediary is unable to observe even the total amount of trades an agent is making with him $\Gamma$ and hence cannot prevent the agent from buying multiples of the contracts he issues.

Not much work exists on convergence to competitive equilibria in economies with asymmetric information. It is noteworthy though that for a particular class of economies with adverse selection $\Gamma$ Biais-Martimort-Rochet (1997) obtain convergence of the equilibria with strategic intermediaries to competitive equilibria with bid-ask spreads also when intermediaries can prevent agents from buying multiple units of the contracts they issue.

The paper is organized as follows. In the next section we describe the economy and define competitive equilibria as well as the equilibria of the game played by financial intermediaries. Convergence is analyzed in section 3. It is first shown that convergence holds when information is symmetric (Proposition 1) as well as in the case of complete observability of trades (Proposition 2) and no observability of trades (Proposition 3). The following result (Propositions 4) shows that $\Gamma$ when information over trades allows intermediaries to prevent multiple trades by the same agent $\Gamma$ convergence to competitive equilibria with bid ask spreads fails. 


\section{The Economy}

We consider an economy where asymmetric information is of the moral hazard (hidden action) type. There are countably many agents $\Gamma$ all ex-ante identical; agents are indexed by $n \in N$. There are two periods $\Gamma t=0,1$, and a single consumption good. Consumption only takes place at $t=1$. Uncertainty is purely idiosyncratic $\Gamma$ and is described by the collection of random variables $\left(\tilde{s}^{n}\right)_{n \in N}$, assumed to be identically and independently distributed $\Gamma$ with support $S=\{H, L\}$; the realization of $\left(\tilde{s}^{n}\right)_{n \in N}$ is commonly observable. Uncertainty enters the economy via the agents' endowments. The (date 1) endowment of agent $n$ is $\tilde{w}^{n}=w\left(\tilde{s}^{n}\right)$; let $w^{H} \equiv w(H), w^{L} \equiv w(L)$ be the agent's endowment in $\Gamma$ respectively $\Gamma$ the idiosyncratic state $H$ and state $L$. Each agents also undertakes a (possibly) unobservable action - we will refer to it as effort - which affects the probability of the realization of his idiosyncratic shock. There are two possible effort levels $a, b$; let $\pi(e)$ be the probability of the realization $s=H$ given the effort level $e \in\{a, b\}$.

Agents' preferences are represented by a (Von Neumann - Morgenstern) utility function of the following form:

$$
\pi(e) \ln \left(c^{H}\right)+(1-\pi(e)) \ln \left(c^{L}\right)-v(e)
$$

where $\left(c^{H}, c^{L}\right)$ denotes consumption respectively in state $H$ and $L \Gamma c \equiv$ $\left(c^{H}, c^{L}\right)$ Tand $v(e)$ denoted the disutility of effort $e .^{3}$

We assume that:

$$
\pi(a)>\pi(b), \quad v(a)>v(b), \quad w^{H}>w^{L}>0
$$

so that $a$ is the 'high' effort and $H$ is the 'good' state.

Let $\Omega$ be the set of parameter values $\left(v(a), v(b), \pi(a), \pi(b), w^{H}, w^{L}\right)$ of the economy which satisfy the above assumptions and the additional condition that at the incentive constrained Pareto optima (see Bisin-Guaitoli (1998) for the standard definition) agents undertake the high effort level $\Gamma=a$.

\subsection{Competitive Equilibria}

Competitive equilibria are characterized by the following market structure: every agent has access to a complete set of pure contingent claims conditional

\footnotetext{
${ }^{3}$ The assumption of logarithmic utility is included just to simplify the calculations in the proof of Lemma 1 and Proposition 4, but is by no means essential.
} 
on his idiosyncratic uncertainty. In particularTand without loss of generality $\Gamma$ two securities are available for trade $\Gamma$ the first paying off one unit of the consumption good in state $H$ Tand the second paying off one unit in state $L$.

In the benchmark case of symmetric information (when the effort level $e$ chosen by each agent is observed) the definition of competitive equilibrium can be written as follows. Let $q^{e}(s)$ denote the (linear) price of the security paying off in state $s$ for agents choosing effort $e .^{4}$

Definition $1 A$ competitive equilibrium with symmetric information ${ }^{5}$ is given by prices $\left(q^{e}(s), e=a, b, s=H, L\right)$, allocations and effort $\left(c^{H}, c^{L}, e\right)$, such that:

(i) $\left(c^{H}, c^{L}, e\right)$ solves the agent's optimization problem

$$
\begin{gathered}
\max _{\left(c^{H}, c^{L}\right) \in \Re_{+}^{2}, e \in\{a, b\}} \pi(e) \ln \left(c^{H}\right)+(1-\pi(e)) \ln \left(c^{L}\right)-v(e) s . t . \\
q^{e}(H)\left(c^{H}-w^{H}\right)+q^{e}(L)\left(c^{L}-w^{L}\right)=0
\end{gathered}
$$

(ii) markets clear $^{6}$ :

$$
\pi(e)\left(c^{H}-w^{H}\right)+(1-\pi(e))\left(c^{L}-w^{L}\right)=0
$$

Both in the budget constraint and in the market clearing condition we have used the fact that the level of trade in the security which pays off is state $s \in\{H, L\}$ equals the excess demand in the same $\operatorname{state} \Gamma\left(c^{s}-w^{s}\right)$.

For economies with asymmetric information (i.e. effort $e$ is unobservable) $\Gamma$ we will consider two cases with regard to the possible forms of the pricing functional.

\footnotetext{
${ }^{4} \mathrm{By}$ allowing the prices of the securities whose payoff is contingent to idiosyncratic uncertainty to depend on the effort level, we effectively allow agents to trade a complete set of securities with payoff contingent on the uncertainty and the effort level.

${ }^{5}$ We limit our attention here to symmetric equilibria. On the other hand, if the agents' choice problem is not convex (as later for economies with asymmetric information), symmetric equilibria may not exist. In such cases we will have to exploit the large number of agents to convexify, allowing for the possibility that agents will make different choices at equilibrium; the definition of competitive equilibria can be easily extended to these situations, at only notational costs.

${ }^{6}$ As standard in these set-ups, we appeal to the law of large numbers to identify $\pi(e)$ with the fraction of agents whose endowment realization is $w^{H}$.
} 
The first is the case in which prices allow for a bid-ask spread but are otherwise linear. To define competitive equilibria in this set-up some additional notation is needed. Let $q^{+}(H)$ and $q^{-}(H)$ denote respectively the buying and selling price of the security paying off if state $H$ occurs; $q^{+}(L)$ and $q^{-}(L)$ denote the buying and selling prices of the security paying off if state $L$ occurs. Let $(x)^{+}$denote $\max (0, x)$ and $(x)^{-}$denote $\min (0, x)$.

Definition 2 A competitive equilibrium with asymmetric information and bid-ask spreads is given by a pair of (bid and ask) prices for each of the two states, $\left(q^{+}(s), q^{-}(s), s=H, L\right)$ and a vector $\left(c^{H}, c^{L}, e\right)$, such that:

(i) $\left(c^{H}, c^{L}, e\right)$ solves the agent's optimization problem

$$
\max _{\left(c^{H}, c^{L}\right) \in \Re_{+}^{2}, e \in\{a, b\}} \pi(e) \ln \left(c^{H}\right)+(1-\pi(e)) \ln \left(c^{L}\right)-v(e) \text { s. } t .
$$

$q^{+}(H)\left(c^{H}-w^{H}\right)^{+}+q^{-}(H)\left(c^{H}-w^{H}\right)^{-}+q^{+}(L)\left(c^{L}-w^{L}\right)^{+}+q^{-}(L)\left(c^{L}-w^{L}\right)^{-}=0$

(ii) markets clear:

$$
\pi(e)\left(c^{H}-w^{H}\right)+(1-\pi(e))\left(c^{L}-w^{L}\right)=0
$$

The second case we consider with regard to the form of the pricing functional is the case in which arbitrary non-linear prices are allowed. Let $q\left(c^{H}-w^{H}, c^{L}-w^{L}\right)$ denote a general non-linear function mapping trades into their value. We then have:

Definition $3 A$ competitive equilibrium with asymmetric information and non-linear prices is given by a map $q: \Re^{2} \rightarrow \Re$ and a vector $\left(c^{H}, c^{L}, e\right)$, such that:

(i) $\left(c^{H}, c^{L}, e\right)$ solves the agent's optimization problem

$$
\begin{gathered}
\max _{\left(c^{H}, c^{L}\right) \in \Re_{+}^{2}, e \in\{a, b\}} \pi(e) \ln \left(c^{H}\right)+(1-\pi(e)) \ln \left(c^{L}\right)-v(e) s . t . \\
q\left(c^{H}-w^{H}, c^{L}-w^{L}\right)=0
\end{gathered}
$$

(ii) markets clear:

$$
\pi(e)\left(c^{H}-w^{H}\right)+(1-\pi(e))\left(c^{L}-w^{L}\right)=0
$$




\section{$2.2 \quad$ Strategic Equilibria}

We present here the strategic equilibrium notion we shall consider in this paper. Assume now the economy is also populated by $I$ financial intermediaries. Each intermediary $i=1, \ldots, I$ can issue $J^{i}$ contracts (securities) $\Gamma$ indexed by $j^{i}=1, \ldots, J^{i} ; J^{i}$ is assumed given $\Gamma$ and large. ${ }^{7}$ Let $J \equiv \sum_{i} J^{i}$; also $\Gamma$ we let $J, J^{i}$ denote the sets of contracts which can be issued as well as their cardinality. A contract is identified by a vector of (possibly negative) payoffs paid by the intermediary to the buyer of the contract $\Gamma$ conditionally on the realization of the publicly observable characteristics of the agent trading the contract. More precisely $\Gamma$ when effort is unobservableГa contract $j$ is a pair $d^{j}=\left(d^{j, H}, d^{j, L}\right)$ describing the payoff respectively in state $H$ and $L{ }^{8}$

Given the set of contracts issued by all intermediaries $\Gamma$ agents choose which contracts to enter and which effort level to undertake; their consumption level is then uniquely determined by their choice of contracts. Perfectly anticipating the agents' choices $\Gamma$ as a function of the set of contracts available to them $\Gamma$ intermediaries strategically choose which contracts to issue $\Gamma$ so as to maximize profits.

With regard to the information available to intermediaries over agents' trades three cases will be considered:

1. Non observability of trades: each intermediary cannot observe any of the trades an agent makes with other intermediaries $\Gamma$ nor whether agents engage in re-trading fractions or multiples of the contracts they purchased (or sold) from them. Thus intermediaries can only separate the buying and selling positions of each agent in their own contracts.

2. Non observability of other trades: each intermediary cannot observe any of the trades an agent makes with other intermediaries $\Gamma$ nor whether agents engage in re-trading fractions of the contracts they purchased (or sold) from them. Thus intermediaries can in this case also impose an upper bound on the trades of each agent in their own contracts.

\footnotetext{
${ }^{7}$ It should be clear from the proof of all the results that the condition that each intermediary can only issue finitely many contracts is never a restriction.

${ }^{8}$ We assume that the trivial contract with zero payoff is always an available choice to the intermediaries. Thus the fact that they are required to issue no less than $J^{i}$ contracts is clearly not restrictive.
} 
3. Complete observability of trades: each intermediary is able in this case to perfectly monitor all the transactions an agent makes $\Gamma$ and hence to implement exclusive contracts. Each agent can only choose to buy one of the $J$ contracts available to him.

It is notationally convenient to define equilibria for asymmetric information economies first. In the case of no observability of trades $\Gamma$ letting $\left\{\lambda_{j^{i}}\right\}_{j^{i} \in J^{i}, i \in I}$ denote the agent's portfolio choices $\Gamma$ the problem solved by agents $\Gamma$ given the set of contracts $d \equiv\left(d^{j^{i}}\right)_{j \in J^{i}, i \in I}$ issued by intermediaries $\Gamma$ can be formally described as follows:

$$
\begin{gathered}
\max _{\lambda \in \Re_{+}^{J}, e \in\{a, b\}, c \in \Re_{+}^{2}} \pi(e) \ln \left(c^{H}\right)+(1-\pi(e)) \ln \left(c^{L}\right)-v(e) \text { s. t. } \\
c^{s}=w^{s}+\sum_{j^{i} \in J^{i}, i \in I} \lambda_{j^{i}} d^{j^{i}, s}, s \in\{H, L\}
\end{gathered}
$$

Note that the portfolio choices of each agent are restricted to be non-negative. This is without loss of generality because the intermediaries can distinguish the buying and selling positions of each agent $\Gamma$ and selling positions can also be described as buying positions of contracts with negative payoffs.

The optimization problem faced by each intermediary $i \in I$, given $\left(d^{i^{i^{\prime}}}\right)_{j^{i^{\prime}} \in J^{i^{\prime}}}, i^{\prime} \neq$ $i$, is then:

$$
\max _{\left(d^{j^{i}}\right)_{j^{i} \in J^{i}} \in \Re^{2 J^{i}}}\left\{-\sum_{j^{i} \in J^{i}}\left[\pi(e) d^{j^{i}, H}+(1-\pi(e)) d^{j^{i}, L}\right] \lambda_{j^{i}}\right\} \text { s. t. }
$$

$$
\left(e,\left(\lambda_{j^{i}}\right)_{j^{i} \in J^{i}}\right) \text { solves problem }(P) \text { when } d=\left[\left(d^{j^{i}}\right)_{j^{i} \in J^{i}},\left(d^{j^{i^{\prime}}}\right)_{j^{i^{\prime} \in J^{i^{\prime}}}}, i^{\prime} \neq i\right]
$$

Thus intermediaries play a simultaneous game in which the choice variable is the menu of contracts they issue Tand perfectly anticipate the agents' choices as a function of the set of contracts issued. ${ }^{9}$

Definition 4 An equilibrium with strategic intermediaries and no observability of trades is then an array $\{(\lambda, e, c), d\}$ such that:

(i) $(\lambda, e, c)$ solves problem $(P)$ given $d$,

(ii) $\left(d^{j^{i}}\right)_{j^{i} \in J^{i}}$ solves (7) given $\left(d^{j^{i^{\prime}}}\right)_{j^{i^{\prime} \in J^{i^{\prime}}}}, i^{\prime} \neq i$.

\footnotetext{
${ }^{9}$ If the consumers' choice, after the intermediaries have decided which contracts to issue, is viewed as a subgame, the strategic equilibrium concept we use is then a subgame perfect Nash equilibrium.
} 
In the case of no observability of other trades $\Gamma$ the agents' problem $\Gamma$ given the set of contracts $d \equiv\left(d^{j^{i}}\right)_{j \in J^{i}, i \in I}$ issued by intermediaries $\Gamma$ is:

$$
\begin{gathered}
\max _{\lambda \in[0,1]^{J}, e \in\{a, b\}, c \in \Re_{+}^{2}} \pi(e) \ln \left(c^{H}\right)+(1-\pi(e)) \ln \left(c^{L}\right)-v(e) \text { s. t. } \\
c^{s}=w^{s}+\sum_{j^{i} \in J^{i}, i \in I} \lambda_{j^{i}} d^{j^{i}, s}, s \in\{H, L\}
\end{gathered}
$$

where the agents' portfolio in each security $j$ is restricted to the set $[0,1] \Gamma$ because the intermediary can impose an upper bound (with no loss of generality set equal to one) on trades in its own contracts. ${ }^{10}$ The intermediaries' choice problem is then obtained by a straightforward reformulation of the one above Treplacing $(P)$ with $\left(P^{\prime}\right)$ in $(7)$. The formal definition of an equilibrium with strategic intermediaries and no observability of other trades is similarly obtained from Definition 4 by replacing $(P)$ with $\left(P^{\prime}\right)$.

FinallyГin the case of complete observability of trades $\Gamma$ the agents' problem $\Gamma$ given the set of contracts $d \equiv\left(d^{j^{i}}\right)_{j \in J^{i}, i \in I} \Gamma$ is :

$$
\begin{gathered}
\max _{\lambda \in\{0,1\}^{J}, e \in\{a, b\}, c \in \Re_{+}^{2}} \pi(e) \ln \left(c^{H}\right)+(1-\pi(e)) \ln \left(c^{L}\right)-v(e) \text { s. t. } \\
\lambda_{j^{i}}=1 \Rightarrow \lambda_{j^{\prime i^{\prime}}}=0 \forall j^{\prime i^{\prime}} \neq j^{i} \\
c^{s}=w^{s}+\sum_{j^{i} \in J^{i}, i \in I} \lambda_{j^{i}} d^{j^{i}, s}, s \in\{H, L\}
\end{gathered}
$$

The specification of the intermediaries' choice problem and the formal definition of an equilibrium with strategic intermediaries and complete observability of trades are again obtained by replacing $(P)$ with $\left(P^{\prime \prime}\right)$ respectively in $(7)$ and Definition 4.

For economies with symmetric information $\Gamma$ since effort $e$ is observable $\Gamma$ intermediaries are allowed to index the payoff of the contracts they offer to

\footnotetext{
${ }^{10}$ The set of contracts each intermediary can issue is now larger than in the case of no observability of trades. However, since the number $J^{i}$ of contracts which intermediary $i$ issues has been set arbitrarily large, this situation can be modelled by assuming that, as in the previous case, the intermediary issues $J^{i}$ contracts; however, with no observability of other trades, contracts are characterized not only by their payoff but also by an upper bound on trade in them. The same is true in the other case analyzed next.
} 
$e$. We can then capture the different assumptions on observability of trades with the same restrictions as above on the choice of $\lambda_{j} \Gamma$ for all $j$.

The problem solved by agents $\Gamma$ given the set of contracts $d \equiv\left(d^{j^{i}}\right)_{j \in J^{i}, i \in I}$ issued by intermediaries $\Gamma$ can then be formally described as follows:

$$
\begin{gathered}
\max _{\lambda \in \Lambda, e \in\{a, b\}, c \in \Re_{+}^{2}} \pi(e) \ln \left(c^{H}\right)+(1-\pi(e)) \ln \left(c^{L}\right)-v(e) \text { s. t. } \quad\left(P^{\prime \prime \prime}\right) \\
c^{s}=w^{s}+\sum_{j^{i} \in J^{i}, i \in I} \lambda_{j^{i}} d^{j^{i}, s, e}, s \in\{H, L\}, e \in\{a, b\}
\end{gathered}
$$

where $\Lambda$ corresponds to $\Re_{+}^{J} \Gamma[0,1]^{J} \Gamma\left\{\lambda^{j} \in\{0,1\}, \forall j: \lambda_{j^{i}}=1 \Rightarrow \lambda_{j^{i^{\prime}}}=0, \forall j^{\prime i^{\prime}} \neq j^{i}\right\} \Gamma$ respectively $\Gamma$ for the case of no observability of trades $\Gamma$ no observability of other trades $\Gamma$ and complete observability of trades.

The optimization problem faced by each intermediary $i \in I$, given $\left(d^{j^{i^{\prime}}}\right)_{j^{i^{\prime}} \in J^{i^{\prime}}}, i^{\prime} \neq$ $i$, is then:

$$
\max _{\left(d^{j^{i}}\right)_{j^{i} \in J^{i}} \in \Re^{4 J^{i}}}\left\{-\sum_{j^{i} \in J^{i}}\left[\pi(e) d^{j^{i}, H}+(1-\pi(e)) d^{j^{i}, L}\right] \lambda_{j^{i}}\right\} \text { s. t. }
$$

$\left(e,\left(\lambda_{j^{i}}\right)_{j^{i} \in J^{i}}\right)$ solves problem $\left(P^{\prime \prime \prime}\right)$ when $d=\left[\left(d^{j^{i}}\right)_{j^{i} \in J^{i}},\left(d^{j^{i^{\prime}}}\right)_{j^{i^{\prime} \in J^{i^{\prime}}}}, i^{\prime} \neq i\right]$ and The definition of equilibrium under the different assumptions on observability of trades for economies with symmetric information are a straightforward extension of those for economies with asymmetric information.

\section{Convergence}

We begin by showing that convergence always holds when information is symmetric (i.e. effort is commonly observable) $\Gamma$ for each of the above possible assumptions about the observability of trades.

Proposition 1 When effort is publicly observable, there exists a unique competitive equilibrium, where all agents undertake the high level of effort and fully insure, at fair odds (i.e. the consumption allocations satisfy $\frac{c^{H}-w^{H}}{c^{L}-w^{L}}=$ $\left.-\frac{1-\pi(a)}{\pi(a)}\right) .{ }^{11}$ For I sufficiently large, equilibria with strategic intermediaries,

\footnotetext{
${ }^{11}$ Strictly speaking, there are also other equilibria, with an unfair price (i.e. $\frac{q^{\alpha}(H)}{q^{\alpha}(L)} \neq$ $\left.\frac{\pi(a)}{1-\pi(a)}\right)$ and no trade on the contracts contingent on a high level of effort. Such equilibria though are not robust to any refinement in the spirit of trembling hand perfection (see Gale (1992)).
} 
for all the assumption on observability of trades, are characterized by the same allocations.

The existence (and in fact the Pareto optimality) of such equilibria is immediate given the simple structure of the economy. Convergence also follows by a standard argument. At any equilibrium with strategic intermediaries $\Gamma$ as long as $I \geq 2$, all contracts traded offer full insurance $\Gamma$ conditionally on a high level of effort $\Gamma$ at fair prices. In fact contracts offering full insurance at more than fair prices make negative profits and hence will not be issued. On the other hand $\Gamma$ contracts offering insurance at less than fair prices can never be an equilibrium outcome as $\Gamma$ if they are traded $\Gamma$ a profitable opportunity for undercutting arises. By similar considerations we can show that contracts offering less or more than full insurance at fair prices will not be traded at equilibrium.

When effort is privately observed strategic equilibria with complete observability of trades do converge to competitive equilibria with asymmetric information and non-linear prices.

Proposition 2 There exists a unique, incentive efficient, competitive equilibrium ${ }^{12}$ with non-linear prices where all agents undertake the high level of effort and achieve partial insurance. For I sufficiently large, all equilibria with strategic intermediaries and complete observability of trades also support the same allocation.

Proof. Let $e\left(c^{H}-w^{H}, c^{L}-w^{L}\right)$ be the map describing the agents' optimal choice of effort $\Gamma$ for any possible level of net trades. In the framework of the economy under examination $\Gamma$ this map is well-defined and single-valued (except at one point where agents' are indifferent in the effort choice). Consider then the pricing functional $q\left(e\left(c^{H}-w^{H}, c^{L}-w^{L}\right)\right)$; it is immediate to see that this pricing functional constitutes a competitive equilibrium with nonlinear prices $\Gamma$ and decentralizes the incentive efficient allocation. Turning next to the equilibrium with strategic intermediaries $\Gamma$ by a similar argument as in Proposition 1 Tas long as there are $I \geqslant 2$ intermediaries $\Gamma$ they will always

\footnotetext{
${ }^{12}$ In this case too other equilibria with non-linear prices exist. However, only the incentive efficient equilibrium satisfies a 'trembling hand' refinement; see again Gale (1992).
} 
choose to issue exclusive contracts offering partial insurance at the incentive efficient levelГand inducing a high level of effort.

We will show next that strategic equilibria with no observability of trades do converge to competitive equilibria with asymmetric information and bidask spreads. For this $\Gamma$ we consider first what are the properties of competitive equilibria with bid-ask spreads. It should be clear ggiven the simple structure of the economy and the specification of contracts available to agents $\Gamma$ that competitive equilibria with bid-ask spreads $\Gamma$ if they exist $\Gamma$ can only be of the following three types:

1. equilibria with low effort and full insurance (i.e. $c^{H}=c^{L}=\pi(b) w^{H}+$ $\left.(1-\pi(b)) w^{L}\right)$, purchased at the fair price $q^{-}(H)=\pi(b), q^{+}(L)=1-$ $\pi(b)$;

2. equilibria with no trade and high effort (i.e. $c^{H}=w^{H}, c^{L}=w^{L}, e=a$ );

3. 'mixed' equilibria where a fraction of the agents in the population exert high effort $\Gamma$ while the others exert low effort $\Gamma$ and both buy insurance $\Gamma$ at the same price (i.e. $\left.c^{H}(a)<w^{H}, c^{H}(b)<w^{H}, \frac{w^{H}-c^{H}(b)}{c^{L}(b)-w^{L}}=\frac{w^{H}-c^{H}(a)}{c^{L}(a)-w^{L}}\right)$, though in different amounts.

In Bisin and Gottardi (1997) it is shown that a competitive equilibrium always exists in this set-up $\Gamma$ and a complete characterization is provided of the set of equilibria for the various regions of the parameter space $\Omega$.

Proposition 3 For I sufficiently large, all strategic equilibria with no observability of trades are characterized by the same allocation as competitive equilibria with bid-ask spread.

Again $\Gamma$ the proof follows by a simple 'Bertrand competition' argument.

On the other hand $\Gamma$ we will now show that when there is asymmetric information and no observability of other trades $\Gamma$ convergence to competitive equilibria with bid-ask spread may not hold. In other words $\Gamma$ when intermediaries can impose an upper bound restriction on agents' trades with themselves $\Gamma$ they can exploit this restriction to produce a barrier to the entry of other intermediaries $\Gamma$ thereby obstructing convergence of strategic equilibria to competitive equilibria with bid-ask spreads. 
Note that the intermediaries' ability to construct barriers to entry by exploiting their information on agents' trades vanishes in the limit for the number of intermediaries $I$ tending to infinity $\Gamma$ in economies with symmetric information (as shown in Proposition 1) $\Gamma$ as well as in economies with complete observability of trades (Proposition 2). Moreover $\Gamma$ in the class of economies with adverse selection studied by Bias-Martimort-Rochet (1997) $\Gamma$ barriers to entry also vanish in the limit and the convergence to competitive equilibrium allocations with bid-ask spreads obtain.

To show that convergence may fail in economies with no observability of other trades $\Gamma$ we focus our attention here on the equilibria for a subset of the parameter space:

Lemma 1 There exists an open set of economies $\Gamma \subset \Omega$ such that for any economy in $\Gamma$ the only competitive equilibria with bid-ask spreads are either equilibria with no trade and high effort, or 'mixed' equilibria.

Proof. The proof is immediate. Let $\Gamma=\left\{v(a), v(b), \pi(a), \pi(b), w^{H}, w^{L} \in \Omega\right.$ :

$$
\begin{aligned}
\frac{\pi(b)(1-\pi(a)) w^{H}}{\pi(a)(1-\pi(b)) w^{L}} & <1 \\
v(a)-v(b)-\pi(a) \ln \left(\frac{\pi(a)}{\pi(b)}\right)-(1-\pi(a)) \ln \left(\frac{1-\pi(a)}{1-\pi(b)}\right) & < \\
(1-\pi(a)) \ln \left(\frac{w^{L}(1-\pi(b))}{1-\pi(a)}\right)+\pi(a) \ln \left(\frac{\pi(b) w^{H}}{\pi(a)}\right)-\ln \left(\pi(b) w^{H}+(1-\pi(a)) w^{L}\right) &
\end{aligned}
$$

It is straightforward to check that $\Gamma \neq \emptyset$. The first inequality defining $\Gamma$ implies that $\Gamma$ if agents' effort level is high $\Gamma$ their optimal choice of trade in insurance contracts $\Gamma$ at the prices $q^{-}(H)=\pi(b), q^{+}(L)=1-\pi(b), q^{+}(H)=$ $\pi(a), q^{-}(L)=\pi(a)$, is zero (i.e. no trade). The second inequality then says that $\Gamma$ at these prices $\Gamma$ agents prefer to exert a high level of effort (and hence a zero level of trade in insurance contracts) to a low effort level $\Gamma$ and the optimal level of trades in that case $\Gamma$ i.e. full insurance. Hence for economies in $\Gamma$ competitive equilibria with low effort do not exist. Since in Bisin and Gottardi (1997) we know that competitive equilibria with bidspreads always exist $\Gamma$ we conclude that only no trade equilibria with high effort $\Gamma$ or 'mixed' equilibria exist in this region. 
The following result then shows that for an open set of economies having a non-empty intersection with the set of economies whose competitive equilibria have been characterized in Lemma $1 \Gamma$ and for $I$ large $\Gamma$ no equilibrium with strategic intermediaries and no observability of other trades can be 'close' to any of the competitive equilibria with bid-ask spread $\Gamma$ i.e. that convergence does not hold. More precisely:

Proposition 4 For an open subset of economies no equilibrium with strategic intermediaries and no observability of other trades converges, for $I \rightarrow \infty$, to a competitive equilibrium with bid ask spreads.

\section{Proof.}

The proof is organized in four main steps.

In the first three steps the result is established for the case in which the agents' admissible portfolio choices are restricted to lie in the set $\lambda \in\{0,1\}^{J}$, i.e. agents are not allowed to buy fractions of the contracts issued. Step 1 shows that there is there is an open $\Gamma$ non-empty subset $\Gamma^{\prime} \subset \Omega$ of economies $\Gamma$ for each of which there exists a non-empty set of consumption allocations (different from the endowment point) with the property that agents at those allocations prefer to exert the high effort level and not to trade any contract offering supplemental insurance at the rate $\frac{1-\pi(b)}{\pi(b)}$. Furthermore $\Gamma$ we show that the intersection of $\Gamma^{\prime}$ with the set $Г \Gamma$ characterized in Lemma $1 \Gamma$ is also non-empty and open.

In the rest of the argument it is shown that $\Gamma$ for all economies in $\Gamma^{\prime} \Gamma$ all the Nash equilibria with strategic intermediaries are different $\Gamma$ for $I$ large enough $\Gamma$ both from the no trade equilibria with high effort and the 'mixed' equilibria. Since $\Gamma$ by Lemma $1 \Gamma$ the competitive equilibria with bid-ask spread of all economies in $\Gamma$ can only be of either of these two types $\Gamma$ it follows that $\Gamma$ for all economies in $\Gamma \cap \Gamma^{\prime}$, no strategic equilibrium converges to a competitive equilibrium with bid-ask spreads.

In Step 2 it is shown that for all economies in $\Gamma \cap \Gamma^{\prime}$, a zero level of trade (with high effort) cannot be an equilibrium with strategic intermediaries $\Gamma$ if $I$ is sufficiently large. This will be established by proving that at the no trade allocation there are contracts $\Gamma$ which if offered $\Gamma$ would lead the agents to a strictly preferred allocation (i.e. would be accepted) and would make positive profits. Thus there exists a profitable deviation $\Gamma$ so the no trade allocation cannot be supported as an equilibrium. The complication in the argument 
comes from the fact that we have to show that profitable deviations exist even in the presence of 'latent contracts' $\Gamma$ i.e. non-trivial contracts which are not traded at equilibrium $\Gamma$ but play a role in deterring entry Tor the proposal of other contracts. ${ }^{13}$ In Step 3 it is then shown that the same result is true for the allocation at a 'mixed' equilibrium.

Finally in Step 4 we show that the result obtained above extends to the case in which agents are allowed to trade fractions of the contracts offered $\Gamma$ i.e. their admissible portfolio choices lie in the set $[0,1]$.

Step 1. Let $A$ be the set of pairs $\left(c^{L}, c^{H}\right) \geq 0$ such that

$$
\begin{gathered}
c^{L} \geq w^{L} \\
\frac{1-\pi(a)}{\pi(a)} \leq\left|\frac{c^{H}-w^{H}}{c^{L}-w^{L}}\right| \leq \frac{1-\pi(b)}{\pi(b)}
\end{gathered}
$$

This is a section of a cone with vertex at the endowment point and boundary defined by the two lines $\Gamma$ with slope $\Gamma$ respectively $\frac{1-\pi(a)}{\pi(a)}$ and $\frac{1-\pi(b)}{\pi(b)}$, which go through the endowment point (i.e. the zero profit loci for high and low effort respectively); see Figure 1. All contracts in $A$ such that $\left|d^{j, H} / d^{j, L}\right| \in$ $\left(\frac{1-\pi(a)}{\pi(a)}, \frac{1-\pi(b)}{\pi(b)}\right)$ will make positive profits if $e=a$, negative profits if $e=b$.

Denote then by $B$ the set of pairs $\left(c^{L}, c^{H}\right) \geq 0$ such that

$$
\pi(a) \ln \left(c^{H}\right)+(1-\pi(a)) \ln \left(c^{L}\right)-v(a) \geq \ln \left(\pi(b) c^{H}+(1-\pi(b)) c^{L}\right)-v(b)
$$

This region identifies the set of consumption allocations where agents prefer to exert high effort $\Gamma$ and are not willing to buy additional contracts $\Gamma$ offering them full insurance $\Gamma$ at the rate $(1-\pi(b)) / \pi(b)^{14}$. Rewrite inequality (9) as follows:

$$
\exp \left\{-(v(a)-v(b)\}\left(\frac{c^{H}}{c^{L}}\right)^{\pi(a)} \geq(1-\pi(b))+\pi(b)\left(\frac{c^{H}}{c_{L}}\right)\right.
$$

\footnotetext{
${ }^{13}$ Hellwig (1983) has first shown in a similar set-up how equilibrium allocations with high effort level can be sustained by the presence of 'latent contracts'. See Bisin-Guaitoli (1998) for a characterization of such equilibria.

${ }^{14}$ If the agents were to buy these additional contracts, their optimal choice of effort would obviously be $e=b$.
} 
It can be easily verified that if the following condition holds:

$$
v(a)-v(b)<\pi(a) \ln \left(\frac{\pi(a)}{\pi(b)}\right)+(1-\pi(a)) \ln \left(\frac{w^{L}(1-\pi(b))}{1-\pi(a)}\right)
$$

the set $B$ is a non-empty cone with vertex at the origin. Furthermore $\Gamma$ the boundary of $B$ is given by two rays through the origin with slope $\alpha_{1}, \alpha_{2}$, both strictly greater than one $\Gamma$ which are obtained as solutions of the equation:

$$
\exp \left\{-(v(a)-v(b)\}(\alpha)^{\pi(a)}=(1-\pi(b))+\pi(b)(\alpha)\right.
$$

i.e. $c^{H} / c^{L} \in\left[\alpha_{1}, \alpha_{2}\right] \Rightarrow\left(c^{L}, c^{H}\right) \in B$. See Figure 1 .

Let $\Gamma^{\prime} \subset \Omega$ be defined by the set of parameter values which satisfy (10) as well as the following additional condition:

$$
w^{H} / w^{L}>\min \left\{\alpha_{1}, \alpha_{2}\right\}
$$

It is immediate to see that the set $\Gamma^{\prime}$ is open nnon-empty. Furthermore $\Gamma$ for any economy whose parameter values lie in $\Gamma^{\prime}$ the subsets $A$ and $B$ of the space of allocations described above have a non-empty intersection $\Gamma$ which is different from the singleton set containing only the endowment point $\left\{w^{H}, w^{L}\right\}$. Recalling then the characterization of the set $\Gamma$ given in Lemma $1 \Gamma$ we can also say that for any economy in $\Gamma$ the endowment point is an interior point of the set $B$, so that $A \cap B \neq \emptyset, A \cap B \supset\left\{w^{H}, w^{L}\right\}$; hence any such economy also belongs to the set $\Gamma^{\prime}$, or $\Gamma \subset \Gamma^{\prime}$.

Step 2. We will show first that $\Gamma$ for all economies in $\Gamma \cap \Gamma^{\prime} \Gamma$ there is no equilibrium in which all agents remain at their endowment point (i.e. there is no trade). Since for such economies the endowment point belongs to the interior of $B$ Cat a no trade allocation agents choose effort $a$. At any point of $A \cap B$ (as in any point of underinsurance) the agents' marginal rate of substitution is (in absolute value) greater than $(1-\pi(a)) / \pi(a)$. Hence there are allocations $\Gamma$ in the interior of $A \cap B$, which are strictly preferred by agents so that if no other non-trivial contract is issued $\Gamma$ there are also contracts which if proposed would make positive profits.

To complete the argument for this case we need to show that a profitable deviation exists even if the no trade allocation obtains when 'latent contracts' are issued. Since the endowment point belongs to the interior of $B \Gamma$ any 
contract such that $\bar{d}^{j, H} / \bar{d}^{j, L}=-(1-\pi(b)) / \pi(b)$ is indeed a possible 'latent' contract $\Gamma$ as it satisfies the condition of not being traded at the endowment point. However such contract will also not be traded at any other point in $A \cap B$; thus it cannot deter the profitable deviation described above.

We will show next that no other contract $\Gamma$ i.e. no contract with payoff such that $-\left(\hat{d}^{j, H} / \hat{d}^{j, L}\right) \in\left(\frac{1-\pi(a)}{\pi(a)}, \frac{1-\pi(b)}{\pi(b)}\right)$, is an admissible 'latent' contract. If such contract is such that $w^{H}+\hat{d}^{j, H} \neq w^{L}+\hat{d}^{j, L}$, there always exists another contract $\Gamma$ with payoff $-\left(\tilde{d}^{j, H} / \tilde{d}^{j, L}\right) \in\left(\hat{d}^{j, H} / \hat{d}^{j, L}, \frac{1-\pi(b)}{\pi(b)}+\epsilon\right)$, for some small $\epsilon>0$, such that agents strictly prefer to trade both contract $\left(\hat{d}^{j, H}, \hat{d}^{j, L}\right)$ and $\left(\tilde{d}^{j, H}, \tilde{d}^{j, L}\right)$, and exert the low effort so the intermediaries issuing $\left(\hat{d}^{j, H}, \hat{d}^{j, L}\right)$ would make negative profits (while those issuing $\left(\tilde{d}^{j, H}, \tilde{d}^{j, L}\right)$ make positive profits). ${ }^{15}$ Hence issuing contract $\left(\hat{d}^{j, H}, \hat{d}^{j, L}\right)$ is not an optimal choice of an intermediary. On the other hand if $w^{H}+\hat{d}^{j, H}=w^{L}+\hat{d}^{j, L}$ there is always another contract $\Gamma$ as we already argued above $\Gamma$ supporting an allocation in $A \cap B \Gamma$ which is strictly preferred by the agent; hence issuing such a contract Trather than $\left(\hat{d}^{j, H}, \hat{d}^{j, L}\right)$, would allow the intermediary to obtain higher (positive) profits. Note that this is true whether or not the 'latent' contracts with payoff $\left(\bar{d}^{j, H}, \bar{d}^{j, L}\right)$ are also issued. Moreover $\Gamma$ even if the contract $\left(\hat{d}^{j, H}, \hat{d}^{j, L}\right)$ is 'split' among any finite number ${ }^{16}$ of intermediaries each issuing a fraction of it $\Gamma$ a profitable deviation still exists. Any intermediary issuing a fraction of the contract can in fact attain higher profits by issuing $\Gamma$ instead of this fraction $\Gamma$ a properly selected contract supporting an allocation in $A \cap B$. This completes the argument that contracts with payoffs $-\left(\hat{d}^{j, H} / \hat{d}^{j, L}\right) \in\left(\frac{1-\pi(a)}{\pi(a)}, \frac{1-\pi(b)}{\pi(b)}\right)$ are not admissible 'latent' contracts.

Step 3. We will show next that no equilibrium exists where a fraction of the agents choose a high and the others a low level of effort Tboth buy insurance and the consumption allocations satisfy the condition $\frac{w^{H}-c^{H}(b)}{c^{L}(b)-w^{L}}=\frac{w^{H}-c^{H}(a)}{c^{L}(a)-w^{L}}$ (i.e. they both lie on the same line $\Gamma$ going through the endowment point $\left.\left(w^{H}, w^{L}\right)\right)$. Evidently

\footnotetext{
${ }^{15}$ Take $\left(\tilde{d}^{j, H}, \tilde{d}^{j, L}\right)$ offering positive insurance at a rate slightly higher than $(1-$ $\pi(b)) / \pi(b)$ if $w^{H}+\hat{d}^{j, H}>w^{L}+\hat{d}^{j, L}$, or negative insurance at a rate slightly lower than $(1-\pi(b)) / \pi(b)$ if $w^{H}+\hat{d}^{j, H}<w^{L}+\hat{d}^{j, L}$.

${ }^{16}$ We should point out that also in the limit case, when $I=\infty$, the same conclusion holds, though by a slighly different argument.
} 
effort levels $a, b$ and the associated allocations $\left(c^{H}(a), c^{L}(a)\right) \Gamma\left(c^{H}(b), c^{L}(b)\right)$. Moreover $\Gamma$ it must be that $\frac{w^{H}-c^{H}(b)}{c^{L}(b)-w^{L}}=\frac{w^{H}-c^{H}(a)}{c^{L}(a)-w^{L}} \in\left(\frac{1-\pi(a)}{\pi(a)}, \frac{1-\pi(b)}{\pi(b)}\right){ }^{17}$. By essentially the same argument as the one used in Step 2 we can show that if $c^{H}(b) \neq c^{L}(b)$ a profitable deviation (given by a contract offering additional positive or negative $i$ insurance at a rate in the interval $\left(\frac{w^{H}-c^{H}(b)}{c^{L}(b)-w^{L}}, \frac{1-\pi(b)}{\pi(b)}+\epsilon\right), \epsilon>$ 0 ) exists. IfT on the other hand $\Gamma c^{H}(b)=c^{L}(b)$, there is always another allocation which is strictly preferred to $\left(c^{H}(a), c^{L}(a)\right)$ by agents making high effort ${ }^{18}$ Гi.e. a profitable deviation exists in this case too. Hence no equilibrium with both levels of effort chosen exists either.

Step 4. Finally Wwe show that the result extends to the case in which trading of fractions is also allowed. This follows immediately from the observation that none of the profitable deviations we considered requires a minimum indivisible quantity to be purchased by agents. The profitability of a contract depends in fact only on its rate $\left|d^{j, H} / d^{j, L}\right|$, the maximal amount of insurance offered $\Gamma$ and the effort chosen by agents. And any consumption allocation strictly preferred to the original candidate allocation would still be preferred to a convex combination of the two.

As shown by Bisin and Guaitoli (1998) $\Gamma$ an equilibrium with strategic intermediaries (possibly in mixed strategies $\Gamma$ and for some selection of the equilibrium play of the agents) always exists; in particular $\Gamma$ for all economies in $\Gamma \cap \Gamma^{\prime}$ a pure strategy equilibrium with high effort and a nonzero level of trade exists (see also Hellwig (1983)). The reader will realize that the arguments above imply that these strategic equilibria cannot converge $\Gamma$ for the number of intermediaries $I$ going to infinity to degenerate equilibria with no trade or to mixed equilibria with both effort levels chosen.

\footnotetext{
${ }^{17}$ If $\frac{w^{H}-c^{H}(b)}{c^{L}(b)-w^{L}}=\frac{w^{H}-c^{H}(a)}{c^{L}(a)-w^{L}}>\frac{1-\pi(b)}{\pi(b)}$, the contracts would make positive profits, but a profitable deviation exists, given by contracts offering a slightly higher payoff in both states. When the inequality holds in the opposite direction the contracts make negative profits.

${ }^{18}$ If $\left(c^{H}(a), c^{L}(a)\right) \notin A \cap B$, agents strictly prefer to buy additional insurance, at a rate $\frac{1-\pi(b)}{\pi(b)}$. On the other hand, when $\left(c^{H}(a), c^{L}(a)\right) \in A \cap B$ it is always possible, since $\frac{w^{H}-c^{H}(a)}{c^{L}(a)-w^{L}}<\frac{1-\pi(a)}{\pi(a)}$, to move slightly upward and to the right in the region $A \cap B$, and find so an allocation strictly preferred to $\left(c^{H}(a), c^{L}(a)\right)$.
} 


\section{Conclusion}

In a simple economy with moral hazard we have considered different assumptions on the observability of agents' trades and examined $\Gamma$ for each of them $\Gamma$ whether the associated competitive equilibria can be obtained as the limit $\Gamma$ as the number of intermediaries gets large of the Nash equilibria of the economy where strategic financial intermediaries compete by issuing contracts. While with symmetric information and with complete observability of trades convergence always holds $\Gamma$ we have shown that the same is not true when the observability of agents' trades is more limited.

\section{References}

BennardoГA. and P.A. Chiappori (1998): 'CompetitionГPositive Profits and Market Clearing under Asymmetric Information' Tmimeo.

BiaisГВ.ГD. Martimort and J.C. Rochet (1997): 'Competing Mechanisms in a Common Value Environment'Tmimeo.

BisinГА.ГЈ. GeanakoplosГР. GottardiГЕ. Minelli and H. Polemarchakis (1998):

'Markets and Information'Tmimeo.

BisinГA. and P. Gottardi (1997): 'Competitive Equilibria of Economies with Moral Hazard: a Note on Helpman-Laffont (1975)' $\Gamma$ mimeo.

BisinГA. and P. Gottardi (1998): 'Competitive Equilibria with Asymmetric Information'Tmimeo.

BisinГA. and D. Guaitoli (1998): 'Inefficiency of Competitive Equilibrium with Asymmetric Information and Financial Intermediaries' $\Gamma$ mimeo.

DubeyГР.ГJ. GeanakoplosГand M. Shubik (1995): 'Default and Efficiency in a General Equilibrium Model with Incomplete Markets' Tmimeo.

GaleГD. (1992): 'A Walrasian Theory of Markets with Adverse Selection' $\Gamma$ Review of Economic StudiesГ59Г229-55. 
HellwigTM. (1983): 'On Moral Hazard and Non-Price Equilibria in Competitive Insurance Markets' $\Gamma$ mimeo.

LisboaГM. (1996): 'Moral Hazard and Nonlinear Pricing in a General Equilibrium Model' $\Gamma$ mimeo.

Prescott $\Gamma$ E. and R. Townsend (1984): 'Pareto Optima and Competitive Equilibria with Adverse Selection and Moral Hazard' $\Gamma$ Econometrica $552 \Gamma$ $21-45 \Gamma$

RadnerГR. (1968): 'Competitive Equilibrium Under Uncertainty' $\Gamma$ EconometricaГ36Г31-58. 